\title{
The New Promising Oleaginous Yeast for Biodiesel Production
}

\author{
Ayșenur YAZICI, Serkan ORTUCU, Mesut TASKIN
}

\begin{abstract}
The objective of this research was selection of a strain the best lipid producing yeast strain and molecular identification for biodiesel production. Soil samples taken from different localities of Erzurum city (Turkey) were used as isolation source. Isolation of lipid producing yeast strains was performed on agar medium. After 48-h incubation, yeast colonies developing on the
\end{abstract} medium were selected and purified. The isolated strains were then screened in order to find out the most potent lipid producing strain. For the determination of lipid concentration, the extraction of total lipids was performed with chloroform-methanol $(2: 1, \mathrm{v} / \mathrm{v})$ mixture. Then, analysis of fatty acids composition of lipids was performed by GC-MS system equipped with a HP-88 capillary column. Fatty acid methyl esters (FAMEs) mix C8-C24 was used as a standard. The FAME peaks were identified and quantitated by comparing the unknown responses to those generated from known standards. The identification of the best lipid producing yeast strain was performed using ITS rDNA analysis and supported by VITEK 2 system. The primers used for the polymerase chain reaction (PCR) were ITS1 (5'-TCCGTAGGTGAACCTGCGG-3') and ITS4 (5'-TCCTCCGCTTATTGATATGC-3'). After PCR, ITS rDNA gene was sequenced in both directions with ITS 1 and ITS 4 primers at Medsantek Co., Ltd., Turkey. Sequences chromatograms were assembled into one complete sequence and the sequence was compared to all known sequences in the Genbank by use of BLASTN 2.2.26+ program. Lipid content of the best lipid producing yeast strain was found $40 \%$ and identified as Rhodotorula glutinis. The major fatty acids of the yeast were palmitic acid (C16:0) $(19.2 \%)$, oleic acid (C18:1) $(46.1 \%)$ and linoleic acid (C18:2) (16.9\%). The most prominent fatty acid was oleic acid. Total content of $\mathrm{C} 16$ and $\mathrm{C} 18$ fatty acids was determined as $96.8{ }^{\circ} \mathrm{C}$. Considering the knowledge that $\mathrm{C} 16$ and $\mathrm{C} 18$ fatty acids are more suitable for biodiesel production, it is possible to say that local isolate $R$. glutinis biomass may be used as a good feedstock for biodiesel production.

Keywords - Oleaginous Yeast, Biodiesel Production, Rhodotorula glutinis

Ayşenur YAZICI

Erzurum Technical University,

Turkey

Serkan ORTUCU

Erzurum Technical University,

Turkey

Mesut TASKIN

Atatürk University,

Turkey 